\title{
Improved visualisation of the design space using nested performance charts
}

Stuart Burgess*, Damiano Pasini and Kazem Alemzadeh

Department of Mechanical Engineering

University of Bristol

Queen's Building

University Walk

Bristol

BS8 1TR, UK

* Corresponding author

Email: s.c.burgess@bris.ac.uk

Tel: 44 (0) 1179288075 


\section{Improved visualisation of the design space using nested performance charts}

Performance charts are an important visual means by which designers explore the design space and optimise the performance of products and systems. Traditional performance charts are usually limited to one or two design variables. However, many design problems have more than two important design variables. This paper presents a new concept of performance chart that can plot the performance of a product or system as a function of more than two design variables. The paper illustrates the new type of chart with the example of the design optimisation of a large mechanical structure that has four design variables.

Keywords: Performance charts, design space, design variables, design team 
Products and systems contain design variables such as dimensions, tolerances and material properties. One of the tasks of the design team is to explore the design space and to select the design variables that give the best performance in terms of technical specification, cost and schedule. Designers often model and analyse the performance of products and systems with physical equations. However, it is generally not possible for designers to optimise and select design variables by just inspecting the bare equations because the equations are often complicated and difficult to directly interpret.

In order to understand and interpret the equations of performance, designers often produce performance charts where aspects of performance are plotted against the design variables. These charts enable designers to visualise how performance changes as a particular design variable is changed. The performance charts are used to select and optimised each design variable.

Traditional performance charts generally only contain one or two design variables. For example, in the area of mechanical design it is common to find performance charts with one $^{1,2,3,4}$ and two $^{5}$ design variables. In standard optimisation analysis, performance charts are also limited to one or two variables ${ }^{6}$. However, products and systems often contain more than two important design variables. In order to graphically represent the performance of a system as a function of more than two design variables it is usually necessary to produce a large number of separate performance charts. These can be difficult to interpret because the design team must understand the relationship between all the separate charts. 
This paper presents a new concept of performance chart called 'nested performance charts' which are able to plot more than two design variables on one single performance chart. The nested performance charts were developed in consultation with an engineering company specialising in the design of large mechanical structures for bulk material handling ${ }^{7}$. The paper illustrates the new performance chart concept with a structural design optimisation problem that contains four design variables.

\section{Case study: Mechanical structure with four design variables}

Figure 1 shows a schematic of a mechanical structure that was studied at the collaborating company ${ }^{8}$. The structure has two lateral beams that are supported by a central pillar and cable stays. The two lateral beams have a uniformly distributed load on them as shown. This type of structure is common in material handling equipment and such structures can weigh several thousand tonnes. The structure has four main design variables. The main design variables are the angles $\alpha$ and $\theta$ and the heights $H_{l}$ and $H_{r}$, as shown in Figure 1 .

[INSERT Figure 1 Mechanical structure with four design variables]

One of the key design goals for the structure shown in Figure 1 is that of minimising the total mass of the system. A low-mass design has the advantages of lower material costs, lower operational costs, lower transport costs and lower environmental impact. However, the designers must also consider the cost of manufacture and time to delivery. If the minimum weight structure is difficult and expensive to manufacture then it is common to choose a configuration which is slightly sub-optimal from the point of view of mass but which is more convenient to manufacture. In practice, the designers consider a range of 
low-mass designs and, in consultation with the production department, select one that gives the best combination of performance in terms of weight and producibility.

For a given loading, $\mathrm{P}$, the weight of a structure can be determined by calculating, for each element, the minimum cross-sectional areas that are required to support the load without yielding. The weight, $\mathrm{W}$, of the structure shown in Figure 1 is modelled by the following equation:

$W=P \frac{\rho g}{\sigma} \frac{1}{n^{2}} \sum_{i=1}^{n} i\left(\frac{H_{l}}{\sin \beta^{2}}+\frac{L}{\tan \beta}+\frac{H_{l} \sin (\beta+\gamma-\vartheta)}{\sin \alpha \sin \beta \sin (\alpha-\gamma+\vartheta)}+\frac{L \cos (\vartheta-\gamma)}{\sin \gamma \cos \vartheta}+\frac{L \sin \vartheta}{H_{r} \sin \gamma}\left(\left(H_{l}-H_{r}\right)^{2}+\left(\frac{H_{l}}{\tan \alpha}+\frac{H_{r}}{\tan \vartheta}\right)^{2}\right)^{0.5}\right.$

where:

$\beta=-\tan ^{-1}\left(\frac{H_{l} \tan \alpha}{H_{l}-L \tan \alpha}\right) \quad \gamma=-\sin ^{-1}\left(H_{l} \sin (\alpha+\vartheta) / \sin \alpha\left(\left(H_{l}-H_{r}\right)^{2}+\left(\frac{H_{l}}{\tan \alpha}+\frac{H_{r}}{\tan \vartheta}\right)^{2}\right)^{0.5}\right.$

where $P$ is the loading, $\rho$ is the density of the material of the members, $g$ is the gravitational constant, $\sigma$ is the maximum permissible stress and $n$ is the number of members. Note: all structural elements are assumed to be prismatic (constant crosssection).

Equation (1) shows that the weight, W, changes if any of the four design variables are changed. When $\beta$ and $\gamma$ are inserted into Equation (1), the equation is extremely large and complicated. As can be seen in Equation (1), the design variables $\alpha, \theta, H_{l}$ and $H_{r}$ appear in 
many places and so it is impossible to determine optimum values just by inspecting the equation.

One approach to finding the optimum values of the design variables is to use a computational search programme such as a hill-climbing algorithm. However such methods have the following disadvantages:

- $\quad$ they do not provide a visual representation of performance trends

- $\quad$ they do not provide much insight

- they do not facilitate communication within a design team

- $\quad$ they do not necessarily give a wide range of low-mass designs

Another approach to finding optimum values of the design variables is to produce performance charts. The advantage of performance charts is that they give a clear visual representation of performance trends for all or a large part of the design space. The designers can use the charts to identify a range of low-mass designs and also to gain insight into why certain structural features are advantageous. The charts can also be shown to team members who are not experts in structural engineering to illustrate how performance is affected by changes in design parameters. When this is done, performance charts help the whole team to come to an agreement about which concept has the best overall performance. Studies have shown that effective communication of design information amongst team members from different disciplines is an important means of achieving successful concurrent engineering ${ }^{9,10}$. 
The next section gives examples of traditional performance charts for the structure shown in Figure 1.

\section{Traditional performance charts}

\subsection{Performance chart with one design variable}

Figure 2 shows a traditional one-variable performance chart for the structure of Figure 1 where the height $H_{l}$ is the design variable. In this case, the remaining three design variables are fixed with the following values: $\alpha=90^{\circ}, \theta=0, H_{r}=0$. The chart shows the weight of the structure for values of the height $H_{l}$ between 0 and $100 \mathrm{~m}$.

This performance chart makes it possible for the designer to immediately make the following useful observations for the assumed values of $\alpha, \theta, H_{r}$ :

- the minimum weight is achieved for a height of $H_{l}=50 \mathrm{~m}$

- $\quad$ the weight increases significantly for small values of $H_{l}$

- $\quad$ the weight does not vary very significantly for $30<H_{l}<80$

Whilst the performance chart shown in Figure 2 is very useful and clear, it has a drawback in that three design variables are fixed and the chart only reveals a small section of the design space. In order to visualise the performance of the whole design space (i.e. different permutations of all variables) it is necessary to produce a large set of design charts for a number of discrete settings of all the variables. Discretisation is a common method for viewing the design space when there are a significant number of design variables ${ }^{11}$. 
The total number of design charts, $N$, required to cover the whole design space is given by: $N=d^{(n-1)}$ where $n$ is the number of design variables $d$ is the number of discrete levels chosen for the discrete variables

In the case of a four variable design problem $(n=4)$, if design charts were produced for three discrete values $(d=3)$ of each of the discrete variables (three variables) then it would be necessary produce $N=3^{(4-1)}=27$ charts in total to cover all the different permutations of values of the discrete variables. Such a large number of design charts makes it very difficult for the design team to view the whole design space and gain insight.

[INSERT Figure 2 Performance chart with one design variable]

\subsection{Performance chart with two design variables}

Figure 3 shows a more sophisticated type of traditional performance chart that plots performance as a function of two design variables. In this case, $H_{l}$ and $H_{r}$ are the design variables whilst $\alpha$ and $\theta$ are fixed with values of: $\alpha=90^{\circ}, \theta=40^{\circ}$. The structural weight is plotted with lines of constant performance that are analogous to altitude lines on a geographic map. The best performance is given by the peak of the highest hill (or the dip of the lowest valley, depending on how the lines are viewed). Since most people are familiar with maps, most people do not have difficultly in understanding two-variable design charts. 
It should be noted that two-variable performance charts can also be created on threedimensional graphs ${ }^{12}$. In such cases it is not necessary to use contour lines because the performance is represented by a surface that is viewed in three-dimensions.

As with the one variable performance chart, it is necessary for the designer to produce separate design charts in order to view the whole design space. For the case of $n$ design variables, the number of two-variable design charts, $N$, required to cover the whole design space is given by:

$N=d^{(n-2)}$

where $n$ is the number of design variables

$d$ is the number of discrete levels chosen for the discrete variables

In the case of a four variable design problem $(n=4)$, if design charts were produced for three discrete values $(d=3)$ of the discrete variables (two of the variables) then it would be necessary to produce $N=3^{(4-2)}=9$ charts. Even though this is a significant improvement on the number of charts required for single-variable performance charts, there are still a significant number of charts required to view the whole design space. It is difficult to interpret nine separate performance charts because the designer must understand the relationship between all the separate charts.

[INSERT Figure 3 Performance chart with two design variables] 


\subsection{Performance chart with three design variables}

There have been attempts to produce three dimensional performance charts that contain three-dimensional shells of constant performance plotted on a three-axes graph. However, such charts are very complex and difficult to understand. Also, since three-dimensional graphs are not encountered in every day life, people generally find them very difficult to get to grips with. An additional problem with three-dimensional graphs is that even if they can be understood, it is almost impossible to read off the values of the design variables from the axes for a particular level of performance.

\section{A new concept of performance chart: nested performance charts}

The nested performance chart consists of a sub-matrix of charts as shown in Figure 4. This particular nested performance chart shows how performance varies as a function of four variables. Two variables $H_{l}$ and $H_{r}$ are considered at three discrete values and the variables $\alpha$ and $\theta$ can be presented as continuous variables in the format of a traditional twodimensional performance chart.

\section{[INSERT Figure 4 Concept of nested performance chart]}

As shown in Figure 4, nested performance charts consist of a matrix of sub-charts that are nested within one global chart. An important feature of the chart is that each sub-chart is positioned such that the centre of the sub-chart is located at the exact values of the discrete variables $\left(H_{l}\right.$ and $\left.H_{r}\right)$ for that particular sub-chart. This means that each sub-chart is seen in 
its correct context. For a given application of the chart, the user must exercise judgement or trial and error in order to find the most appropriate number of discrete levels for each discrete variable.

The nested performance chart is more helpful than having separate design charts because an individual sub-chart is clearly put into context by being nested within a global chart at the right co-ordinates. The layout also shows how charts are related to each other in the overall scheme. By scanning the chart, it is possible to observe performance trends.

One limitation of nested performance charts is that at least two design variables must be discretised. This means that the charts do not generally reveal the exact optimum values of the design variables. However, the charts enable the designer to quickly identify the approximate values of the optimum design variables. If the designer needs to identify the exact optimum values of the design variables, then they can produce more refined nested charts in the appropriate areas.

When there are four design variables in the design problem, the nested performance chart can be constructed with either two or three variables discretised. These two options are shown in the following sections using the structure of Figure 1 as a case study.

\section{Nested performance chart with two discrete variables}

Figure 5 shows an example of a nested design chart for the structural design example considered in this paper. The discrete variables are $H_{l}$ and $H_{r}$ and these are considered at 
three discrete values. The variables $\alpha$ and $\theta$ are plotted on traditional two-variable performance charts within each box of the matrix.

In the top right-hand corner of each sub-chart a ranking is given from $1^{\text {st }}$ to $9^{\text {th }}$ that ranks the peak performance within each chart. $1^{\text {st }}$ represents a peak performance with the lowest weight structure. Figure 5 also shows a path from the worst peak performance to the best peak performance.

For the nested performance charts shown in Figure 5, the following important observations can be made:

- The best peak performance (lowest weight at optimal point) is achieved when the heights $H_{l}$ and $H_{r}$ are maximised.

- There is a large weight penalty when $H_{l}$ is constrained in height.

- There is also a weight penalty when $H_{r}$ is constrained in height although not so much as for $H_{l}$.

- Each column shows that higher values of $H_{l}$ produce performance contours with shallower slopes (wider spaced contours) along the $\alpha$-axis. Therefore for high values of $H_{l}$ the performance is more sensitive to variations in $\theta$ than variations in $\alpha$.

- Each row shows that higher values of $H_{r}$ makes the position of the optimum point move towards decreasing values of $\alpha$. On the contrary, the position of the optimum point along $\theta$ is not particularly sensitive to the increase of $H_{l}$ in each column. 
These observations are much more sophisticated and useful than the observations that could be drawn from the traditional one and two variable design charts described in Section

2. This example shows that nested performance charts have the potential to improve the visualisation of the design space. Engineers in the collaborative company found this type of chart very useful for gaining insight into the design problem and facilitating discussions within the design team.

[INSERT Figure 5 Nested performance chart with two discrete variables]

\section{Nested performance charts with three discrete variables}

\subsection{Version 1: one variable continuous}

Figure 6 shows a version of the nested performance chart where three design variables are discretised. In this case, the angle $\theta$ is discretised as well as the heights $H_{l}$ and $H_{r}$. Only $\alpha$ is shown as a continuous variable. This type of chart can sometimes be more convenient than the one shown in Figure 5 because the optimum performance is easier to read in each box of the matrix.

As with Figure 5 the performance chart shown in Figure 6 can be used to observe trends in performance. However, space availability can make difficult to display 27 graphs, as shown in Figure 6. In the next section, another version of the nested performance chart is presented which overcomes this drawback.

[INSERT Figure 6 Version I of nested performance chart for three discrete variables] 


\subsection{Version II: one variable at optimum value}

Figure 7 shows another version of the nested performance chart where three design variables are discretised. As in Figure $6, H_{l}, H_{r}$ and $\theta$ are discretised and $\alpha$ is the variable. In addition, 27 boxes are displayed on the chart. However, in this case the graph in each cell is a single colour (or shade) that indicates the optimum value of $\alpha$ for that particular combination of discrete variables. The darker the colour, the lower the weight (better performance) and vice versa. This chart can sometimes be more convenient than the previous nested performance charts because there is a direct indication of performance through the colour of the box.

[INSERT Figure 7 Version II of nested performance chart for three discrete variables]

The chart shown in Figure 7 gives a very straightforward visual representation of performance trends throughout the whole design space. One drawback is that the exact value of the optimum angle $\alpha$ is not immediately shown. However, a computer implementation of the chart could allow this to be easily viewed.

As with the previous nested performance charts, the chart of Figure 7 can be used to see the following patterns of performance:

- The best peak performance is achieved when the heights $H_{l}$ and $H_{r}$ are maximised.

- $H_{l}$ has a more significant impact on the performance than $H_{r}$.

- Increases of $\theta$ from $40^{\circ}$ to $60^{\circ}$, improves the performance. 
It is interesting to note that the interpretation of the results in Figure 7 is analogous to the interpretation of the results of a Design Of Experiments (DOE) exercise ${ }^{13}$ because the effect of changing individual parameters can be systematically examined. If it is necessary to see a finer detail of output than that shown by Figure 7 then the user can produce more detailed performance charts for any box that looks to have the potential for high performance.

\section{Conclusions}

Design charts are an important means by which design teams in all domains visualise the performance of products and systems. This paper has presented a new type of design chart called nested design charts that can present performance as a function of more than two variables. The new type of design chart enables the designer to visualise the whole design space on one single design chart. The design chart helps designers to observe subtle trends in performance as variables are changed. The charts also facilitate discussions within the design team. The method has been applied to a structural design optimisation problem that contains four design variables. The method could be extended in principle to design problems with more than four design variables if there are nests within nests. In such cases, it would be necessary to have a computerised implementation of the concept. 


\section{Acknowledgements}

This work has been carried out under an EPSRC research contract number GR/NO3648. The authors acknowledge the support of Strachan and Henshaw Materials Handling Division.

\section{References}

[1] Shanley F. R., Weight-strength Analysis of Aircraft Structures, $2^{\text {nd }}$ edn, New York, Dover (1960).

[2] Cox H.L., The design of structures of least weight, Oxford Pergamon Press (1965).

[3] Caldwell J.B. and Woodhead R.G., Ship structures: some possibilities for improvement, North East Cost Institution - Inst. Eng. \& shipbuilders - Transaction - vol 89, (1973) $101-120$.

[4] Ashby, M., Material selection in mechanical design, Pergamon Press, (1992).

[5] Pasini D., Burgess S. C., Smith D.J, Performance indices for arbitrary scaled rectangular cross-sections in bending stiffness design. Proc. Instn Mech. Engrs, Part L, Journal of Materials: Design and Applications, (2001) vol. 216. 1-13.

[6] Arora, J., Optimum Design, McGraw-Hill, (1989).

[7] Pasini, D., A new theory for modelling the efficiency of material, shape and form, PhD thesis, Bristol University, 2003.

[8] Burgess S.C., Pasini D., Smith D.J., 2001, Form factors: a design method to support the selection of structural concepts ICED 01, Glasgow, 21-23, (2001) pp. 179-186. 
[9] Riahi, H.R., and Pawar, K.S. Modelling and analysis of communication structures in a CE environment, Advances in Manufacturing Technology IX, Proc. Of the $11^{\text {th }}$ Natiuonal Conf on Manufacturing Research, Leicester UK, Set. (1995) pp 688-692.

[10] Zhang, H.C. and Zhang, D., Concurrent engineering: an overview from manufacturing engineering perspectives, Concurrent engineering, 3(3), (1995) pp 221-236.

[11] Chryssolouris, G., Subramaniam. V. and Lee, M., Use of Extreme Value Theory in Engineering Decision Making, Journal of Manufacturing Systems, Vol 13, No. 4, (1994) 302-312.

[12] Li, W., Hu, J.S. and Cheng, S.W., Robust Design and Analysis for Manufacturing Processes with Parameter Interdependency, Journal of Manufacturing Systems, Vol 21, No. 2 (2002), pp 93-100.

[13] Box, G. and Bisgaard, S., Statistical tools for improving designs, Mechanical Engineering, January (1988), pp 32-40. 


\section{List of captions}

Figure 1 Mechanical structure with four design variables

Figure 2 Performance chart with one design variable

Figure 3 Performance chart with two design variables

Figure 4 Concept of nested performance chart

Figure 5 Nested performance chart with two discrete variables

Figure 6 Version I of nested performance chart for three discrete variables

Figure 7 Version II of nested performance chart for three discrete variables 


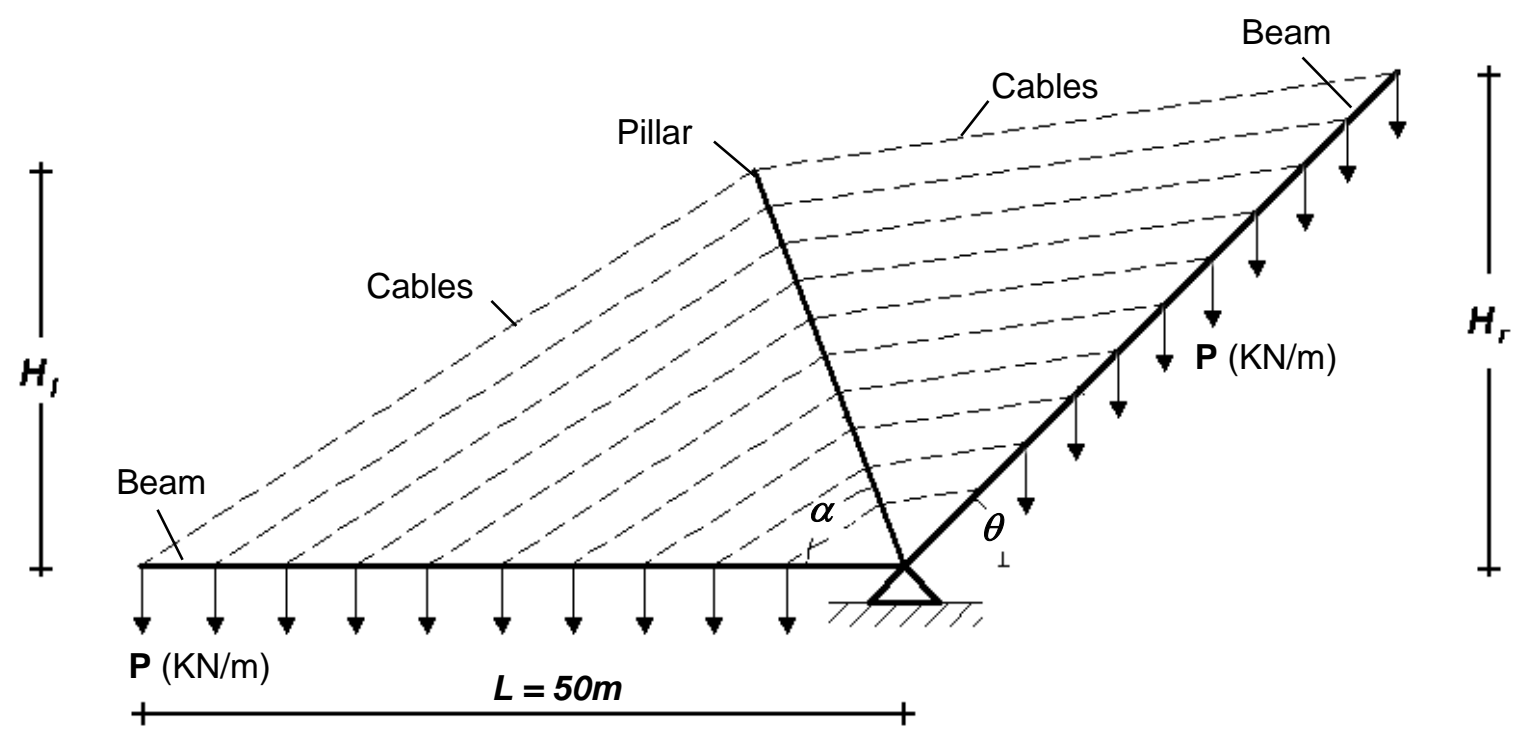

Figure 1 Mechanical structure with four design variables 


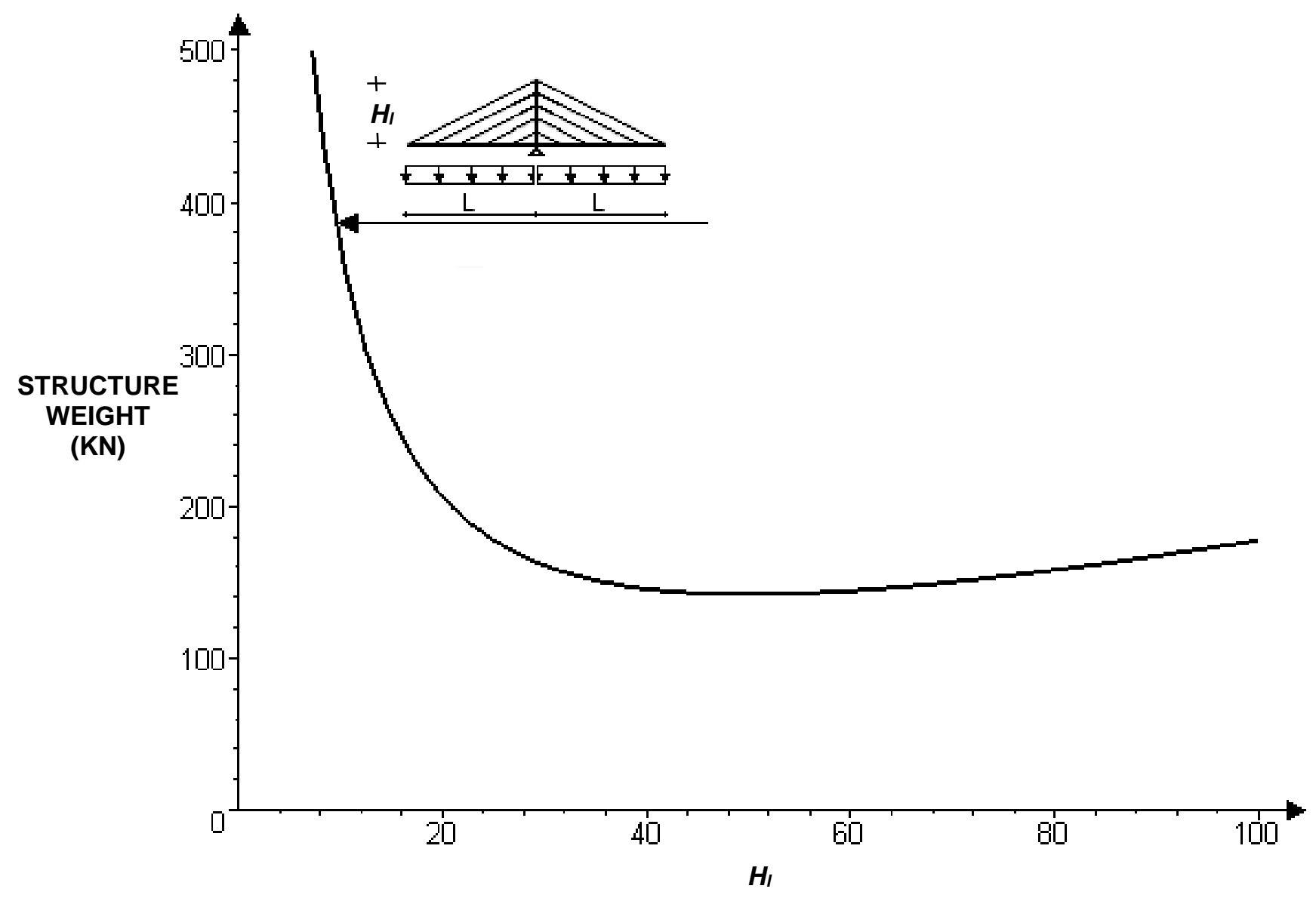

Figure 2 Performance chart with one design variable 


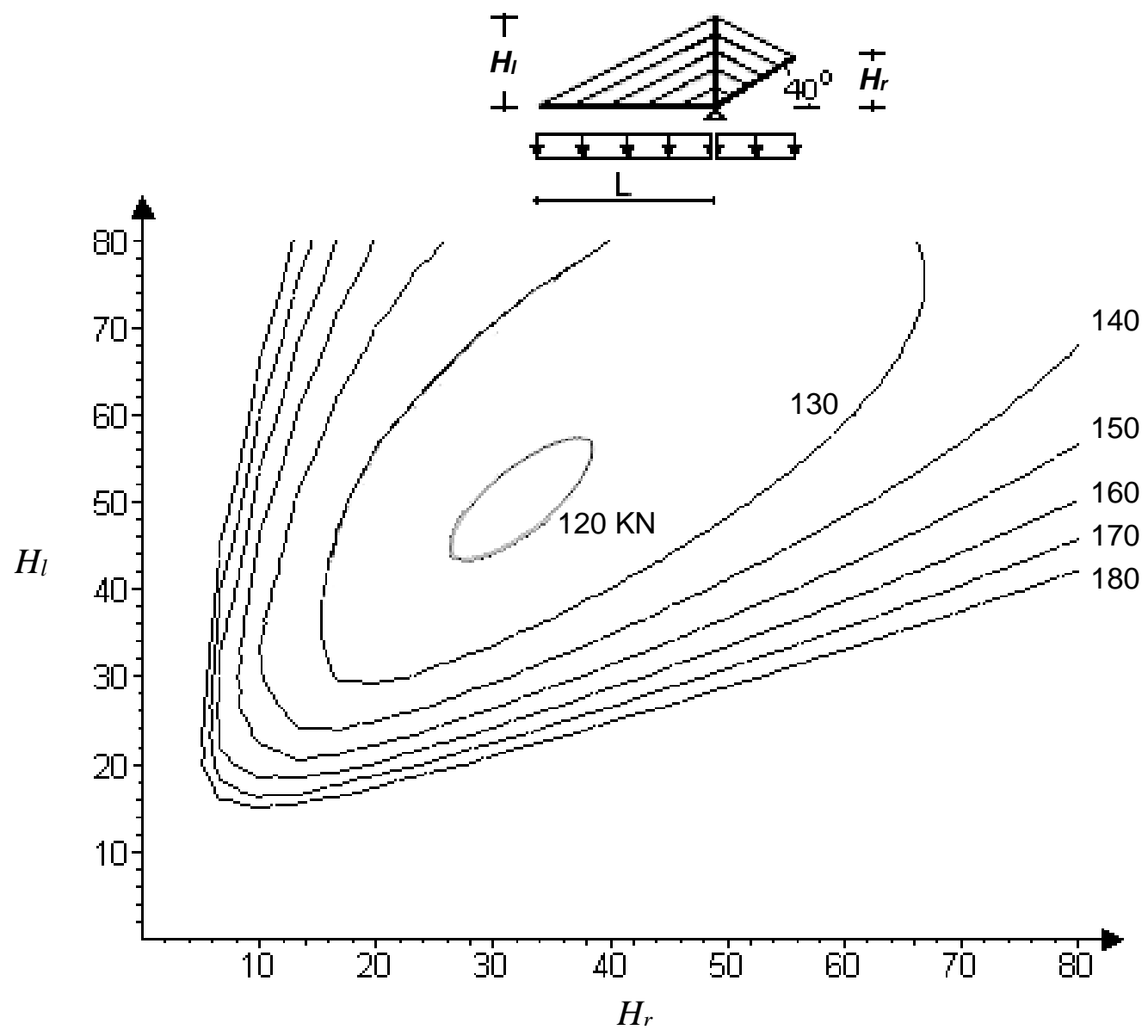

Figure 3 Performance chart with two design variables 


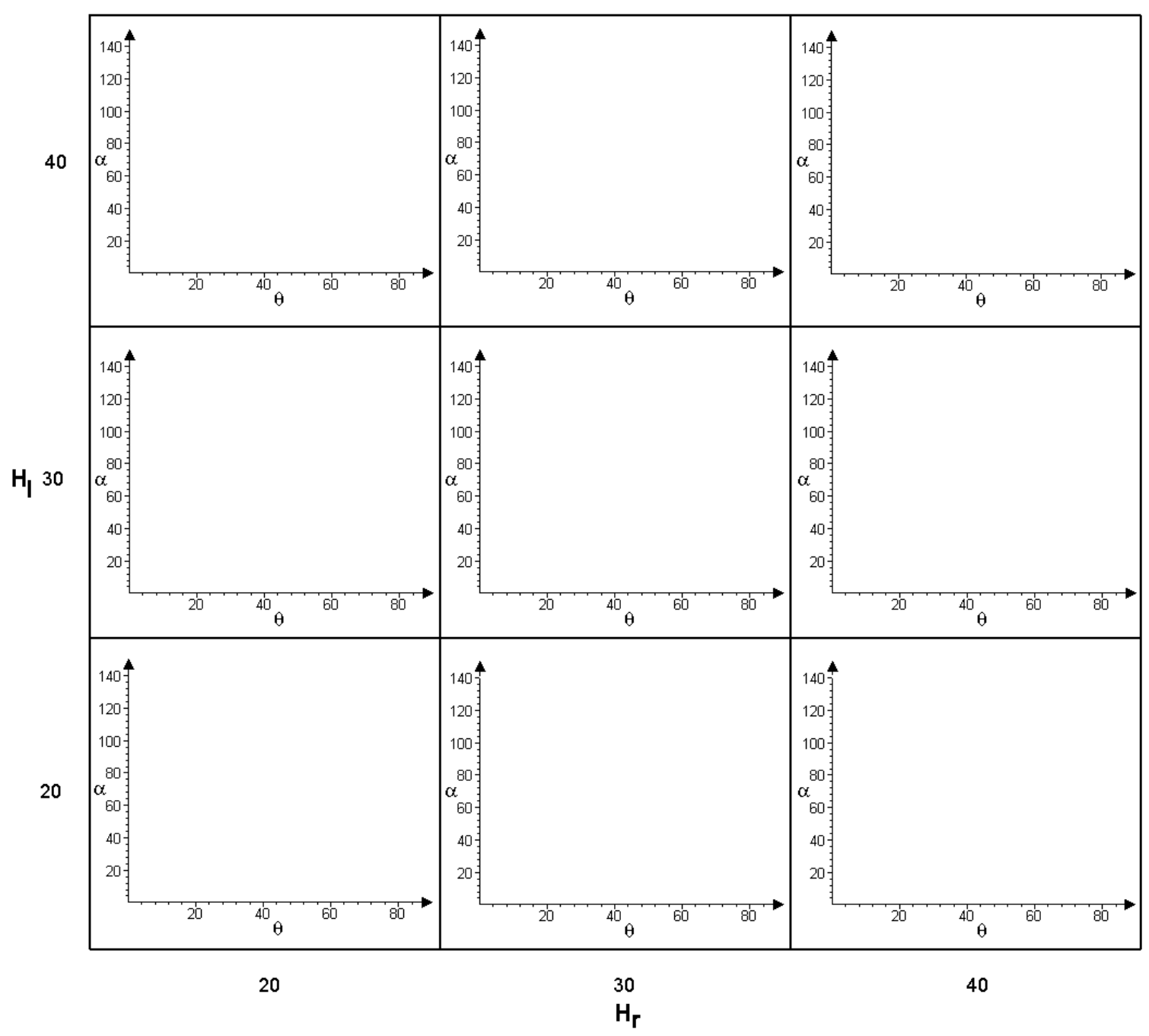

Figure 4 Concept of nested performance chart 

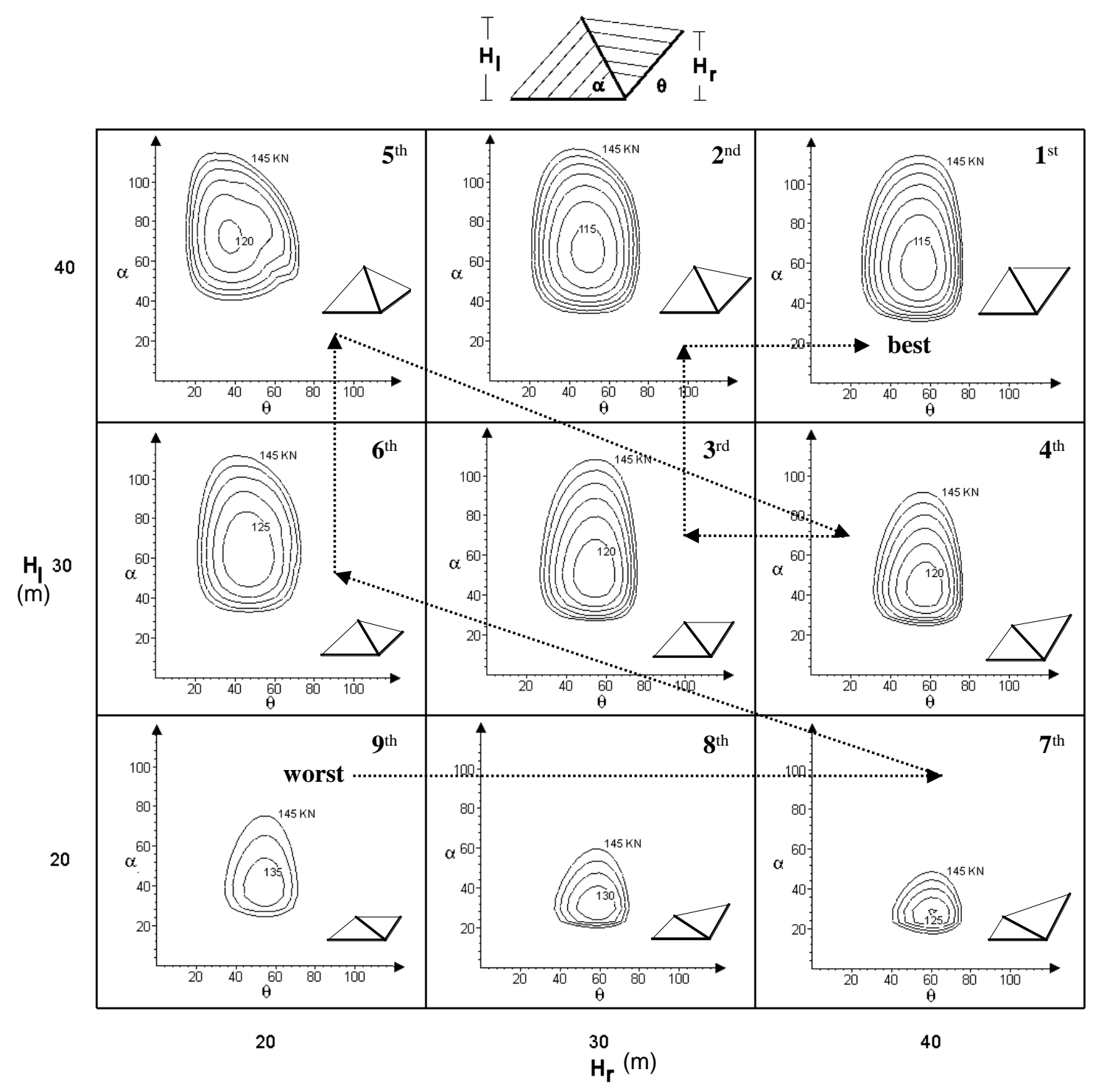

Figure 5 Nested performance chart with two discrete variables 


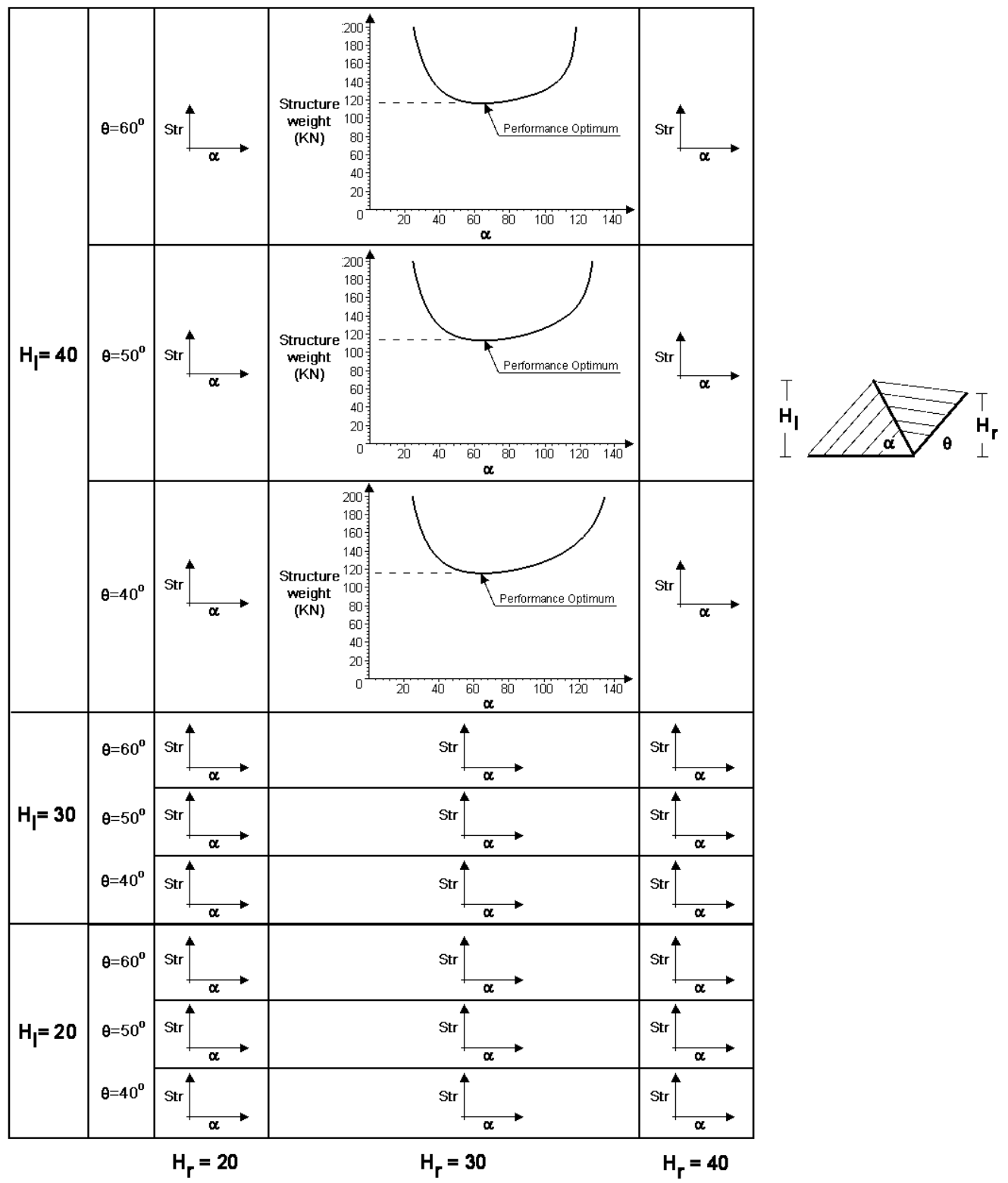

Figure 6 Version I of nested performance chart for three discrete variables 

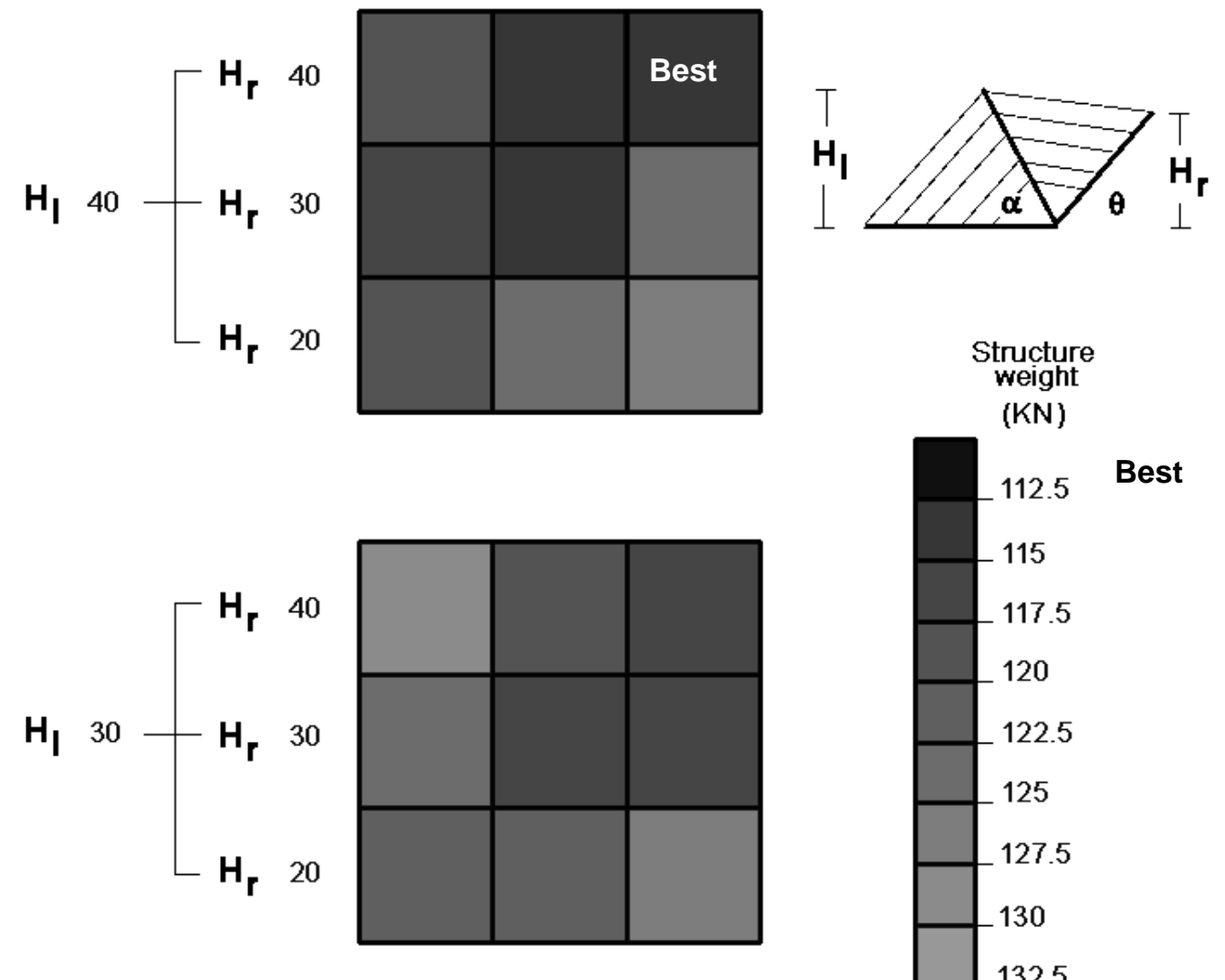

112.5 Best

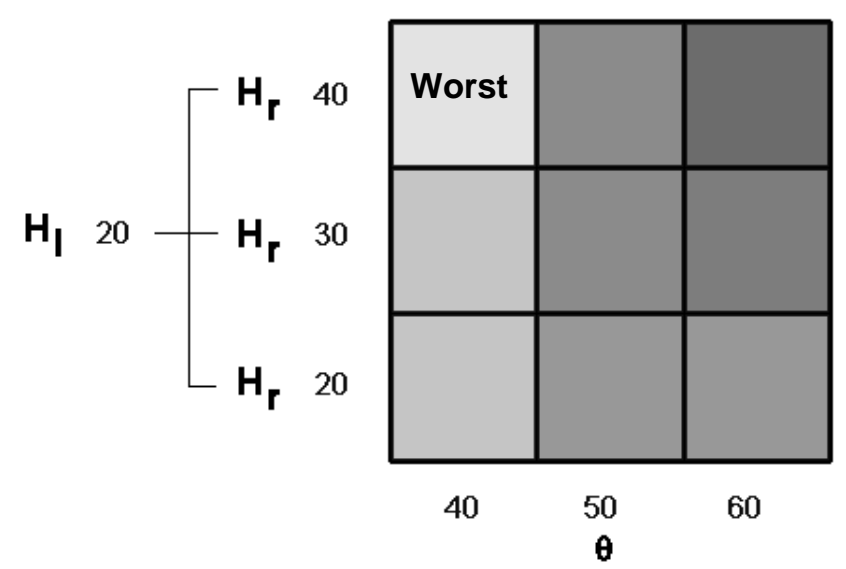

Worst

Figure 7 Version II of nested performance chart for three discrete variables 